\title{
El aprendizaje lúdico de la literatura en niños de educación básica primaria, apoyado en dispositivos tecnológicos como los Ambientes Hipermediales
}

\author{
The playful learning of literature in children of \\ primary school, supported by technological devices as \\ Hypermedial environments
}

\author{
Henry González Martínez ${ }^{1}$ \\ David Jacobo Viveros Granja ${ }^{2}$
}

\section{Resumen}

El presente artículo busca dar a conocer uno de los proyectos de investigación del grupo HIMINI3, titulado: “Impacto de un Ambiente Hipermedial en los procesos de comprensión y producción de texto artístico verbal de estudiantes de grado quinto de educación básica primaria", cuyo objetivo es indagar acerca de los efectos que tiene un Ambiente Hipermedial, producido por este grupo, en el aprendizaje de la literatura de los niños del grado mencionado.

El núcleo artístico del dispositivo es el minicuento o la minificción, y entre los resultados más relevantes se pueden señalar: el progreso en la comprensión y producción de texto artístico breve, la disminución de la brecha digital entre los usuarios estudiantiles y el fomento de una inteligencia colectiva virtual con intención literaria.

\section{Palabras clave}

Ambiente Hipermedial, portal, blog, juego interactivo, minificción.

\section{Abstract}

The present article reports a research project developed by the HIMINI research group, entitled "The Impact of a Hypermedial Environment on the Understanding and Production Processes of Verbal Artistic Texts by Students of Fifth Grade at Primary School". The main aim of this study is to investigate the effects that a hypermedial environment, produced by this research group, may have on the fifth grade students' learning of literature.

The artistic texts that are central to this study are Short-short Stories and flash fiction. The main findings suggest that there is progress in the understanding and production of these types of literary texts, that the digital gap among the students who used the hipermedial environment is reduced, and that it fosters colective virtual intelligence with literary purposes.

\section{Key words}

Hipermedial environment, Webpage, blog, interactive game, flash fiction.

Artículo recibido el 11 de Mayo de 2010 y aprobado el 30 de Agosto de 2010

1 Profesor de tiempo completo e investigador en literatura de la Universidad Pedagógica Nacional de Colombia; Coordinador del Grupo de Investigación en Hipermedia, Minificción, Literatura y Lenguaje, HIMINI. henrygm68@hotmail.com

2 Profesor de tiempo completo de la Universidad Pedagógica Nacional y miembro del Grupo de Investigación en Hipermedia, Minificción, Literatura y Lenguaje, HIMINI. viverosgranja@gmail.com

3 HIMINI: Grupo de Investigación en Hipermedia, Minificción, Literatura y Lenguaje, de la Universidad Pedagógica Nacional de Colombia, reconocido y clasificado en categoría B por COLCIENCIAS. Compuesto por: Henry González, Alfonso Cárdenas, Hilda Mercedes Ortiz, David Jacobo Viveros, Jaime Alejandro Rodríguez, Yuly Viviana Pongutá, Marcela Londoño y Carolina Jiménez. 


\section{Introducción}

El considerable aumento de usuarios, el surgimiento de múltiples redes sociales, la amigabilidad de las utilidades y servicios de Internet, sustentada en bases de datos que los usuarios pueden modificar, tanto en el contenido como en la forma de presentación, vienen adquiriendo fuerza, impulsados por el vertiginoso desarrollo de las tecnologías de la información y la comunicación en los últimos años. Esta amplia gama de servicios y de notables cambios de respuesta en los usuarios, es lo que la crítica ha llamado Web 2.0 (Wikipedia. Web 2.0) ${ }^{4}$ y Web 3.0 (Wikipedia. Web 3.0)5.

Servicios de microblogging (o espacios para remisión de mensajes breves) como Twitter e Identica, por citar sólo dos, permiten a los usuarios, además del uso gratuito, el envío de microtextos (cuya longitud máxima es de 140 caracteres), sea utilizando los sitios Web de estos servicios, el SMS (short message service) o el teléfono celular, y empleando programas como los de mensajería instantánea, Tweetie (López, José, 2010)6, Twinkle (Wikipedia. Twinkle)7, Tweetboard (Frechín, J)8, Facebook

4 En Wikipedia se denomina Web 2.0 a la "segunda generación en la historia del desarrollo de la tecnología Web", caracterizada por el surgimiento de las comunidades de usuarios y una variedad de servicios expresados en las redes sociales, los blogs, los wikis, etc., los cuales "fomentan la colaboración y el intercambio ágil de información entre los usuarios de una comunidad o red social".

5 En nuestros días el neologismo Web 3.0 es utilizado para señalar las transformaciones en el uso y la interacción en la red recurriendo a diferentes caminos, entre los que se incluye la conversión de la red en una base de datos y un movimiento hacia la facilidad en el acceso a los contenidos a través de diversas aplicaciones y otros fenómenos como los siguientes: "el empuje de las tecnologías de inteligencia artificial, la Web Semántica, la Web Geoespacial, o la Web 3D".

6 Tweetie es una aplicación que permite acceder a Twitter y seguir las conversaciones de los contactos de un internauta. Permite ver quién habla de entre los contactos y buscar mensajes sobre un tema o palabra concretos.

7 Twinkle se define como "un conjunto de scripts" para los usuarios que deban llevar a cabo el proceso de mantenimiento de Wikipedia para contrarrestar cualquier información malintencionada. Entre sus funciones se encuentran las siguientes: marcado de artículos, borrado rápido y advertencia a usuarios.

8 Tweetboard se define como "una nueva forma de integrar twitter" en la web para ver los comentarios hechos por otros internautas y escribir las respuestas.
(Wikipedia. Facebook) ${ }^{9}$, TweetDeck ${ }^{10}$ o Twitterrific (http://bitacoras.com/canales/twitterrific) ${ }^{11}$.

Estos servicios se han convertido en un fenómeno social de uso masivo que ha ido creando su propia forma de expresión mediante convenciones propias de los usuarios. En la actualidad se pretende que además de la interacción entre las personas, se puedan utilizar estos desarrollos en campos como el periodismo o la educación, por ello se habla de edutwitters o del periodismo Twitter.

En el caso de Twitter, el ventajoso funcionamiento de este servicio de microblogging en tiempo real permite que la gente se entere incluso de hechos meteorológicos que están ocurriendo en otros lugares del mundo. Bajo la inspiración de estos servicios ya se está pensando en noticias o editoriales que no sobrepasen las 20 palabras, como una alternativa al abarrotado mundo de la información actual.

De este modo, las tecnologías se han convertido en un medio para construir espacios destinados a la comunicación entre amigos, familiares o conocidos; también, en fuentes de información a las que se puede acceder cuando los usuarios han formulado preguntas sobre un tema en la red. Entre las ventajas del microblogging se encuentran: la inmediatez, la precisión en el mensaje para evitar textos confusos, la máxima concentración del lenguaje y el ser un novedoso medio para transmitir información y elaborar textos creativos.

El uso de estas tecnologías en países de Hispanoamérica es todavía muy reducido, sin embargo, la idea de aplicarlas masivamente en la educación es una opción que se está gestando, pues las ventajas que brindan para el aprendizaje en el aula de

9 Facebook se define como un sitio en la web para que el usuario participe a través de redes sociales de forma gratuita; entre sus funciones se encuentran: poder localizar amigos fácilmente, especialmente aquellos con los que por alguna razón se perdió contacto; enviar y recibir mensajes o fotos; $y$ convocar a grupos que tengan un interés común.

10 Según el texto "TweetDeck, la mejor opción para Twittear", TweetDeck se basa en Adobe Air, permite estar enterado de la información de los contactos que tenga el usuario y la información se puede organizar en columnas; su ventaja radica en que puede usarse con Facebook

11 Tweeterrific es una de las aplicaciones de Twitter que facilita ver y seguir más de cerca lo que está ocurriendo en Twitter. Es también de gran utilidad para el uso de comunicaciones mediante iPhone. 
informática y en salones de clase, especialmente equipados, son altamente productivas.

Entre los beneficios que brindan estas tecnologías se observan los siguientes: el docente y el estudiante pueden recurrir a ellas y construir diarios donde se registren los sucesos o experiencias del día acerca de las actividades de la clase; los estudiantes pueden opinar sobre las actividades del día recurriendo a dispositivos tecnológicos; el maestro puede enviar informes a los alumnos acerca del cambio de horario u otro tipo de contenido; además, es posible usar el espacio para producir minicuentos u otro tipo de textos breves en los que se pone a prueba la imaginación y el uso de diseños agradables (Muñoz, 2010).

Alguna tendencia crítica ha opuesto la cultura alfabética a la de la imagen (Do Brito, Marisa, 2009), sin embargo, el computador interactúa con las dos culturas a través de una escritura y una lectura digitales que no atacan el formato impreso, al contrario, agregan posibilidades activas de lectura y escritura para los usuarios. De este modo, la presente investigación desarrolla la cultura alfabética mediante nuevos formatos que ofrecen los dispositivos tecnológicos, otorgándole prioridad a la literatura.

El papel activo del alumno se materializa en los espacios virtuales, porque puede compartir direcciones electrónicas que encuentra en sus búsquedas y que pueden resultar de interés para el resto de participantes (http://www.tecnotic.com/node/746\#); y finalmente, el microblogging permite crear categorías para agrupar los mensajes en etiquetas que aludan a un mismo tema (De Haro, Juan).

Así, las TICs se constituyen como una opción valiosa para llevar a cabo una educación moderna que dialogue con el mundo digital en el que el estudiante se encuentra inmerso. Un docente puede diseñar perfectamente un microblog y utilizarlo para recordar a sus alumnos las labores que deben realizar; también, destinar este espacio para que el alumno consulte links que amplíen las explicaciones recibidas en el aula. Así mismo, los estudiantes pueden registrar sus dudas en el microblog y recibir orientaciones o tutorías.

Las actividades mencionadas permitieron al grupo investigador interrogar los desarrollos de las TICs a favor de objetivos educativos que el docente puede aprovechar, porque una didáctica de la literatura siempre busca renovarse en beneficio del estudiante y del profesor, y una de las vías es a través del apoyo en las TICs, sin querer decir que las Tecnologías de la Información y la Comunicación sean la única opción de renovación de las prácticas en educación, sí se puede afirmar que son en la actualidad una de las más importantes.

En Latinoamérica aún falta aprovechar las ventajas que brindan estas tecnologías para la educación, pues las instituciones todavía no desarrollan el uso de bibliotecas electrónicas que podrían complementar o reemplazar a las que guardan libros y documentos impresos, que tiene ciertas limitaciones de uso y están sujetas al horario escolar.

Este problema podría ser solucionado con un buen empleo de los servicios de Internet, mediante los cuales se puede acceder todo el tiempo a diccionarios, revistas, libros, periódicos, videos, enciclopedias, que se pueden descargar de la red sin ningún costo. El problema se solucionaría, en las escuelas o colegios que no dispongan de estas facilidades, con la instalación de computadores y servicios de Internet dirigidos a facilitar la interacción educativa entre profesores y estudiantes.

Para un niño la posibilidad de acceder a la información desde el aula de informática, desde un café Internet o desde un computador de uso doméstico y con la orientación del profesor, le permite obtener un caudal de conocimientos, navegar por museos, explorar países o enterarse de hechos históricos a través de páginas que recrean sus contenidos por medio del texto, el audio y el video.

La interacción educativa con estas tecnologías contribuye además al desarrollo de habilidades cognitivas (Eduteka, 2007) e intelectuales, y puede preparar al usuario para enfrentarse a la excesiva información que existe en la red. La escuela no solamente debe familiarizar al estudiante con la Internet desde una edad temprana, sino facilitarle condiciones para manejar estrategias de búsqueda y lectura de texto digital que le permitan discriminar y valorar sus contenidos.

Recientemente se ha señalado en el periódico $E l$ Tiempo (2009) que a partir del 2011, el Plan Decenal de Educación requerirá a las instituciones educativas la integración en su currículo de las nuevas tecnolo- 
gías, como parte esencial de los diversos tópicos de aprendizaje. Así mismo, se solicitará a los maestros el diseño de estrategias pedagógicas que estén apoyadas en las TICs.

Además de la escuela, los gobiernos también deben tomar conciencia de la urgencia de integrar al sistema escolar el mundo de las TICs, pero no basta con la dotación de aulas de informática y su conexión a Internet, también es necesario acostumbrar al estudiante a leer y valorar la información obtenida a través de estos medios, disponiendo del tiempo necesario que le permita un óptimo proceso de aprendizaje, pues el contacto reducido o limitado a unas pocas horas semanales no permite observar grandes progresos en el alumno.

Crear una cultura de la tecnología también responde a la necesidad de que no haya un uso irresponsable o inconsciente de la información; si los estudiantes perciben una intención claramente definida en torno al aprendizaje en la interacción con los programas y la información de Internet, asumirán una actitud autónoma y comprometida con su formación, aspecto que puede derivar en la obtención de importantes resultados a corto plazo.

El panorama sobre las tecnologías de la comunicación y la información sugiere múltiples preguntas relacionadas con las formas en que se puede apreciar el progreso de las comunidades educativas en su interacción con las TICs. Uno de estos interrogantes se plantea en torno a la disposición de la institución escolar para los desarrollos tecnológicos actuales: ¿Están o no dispuestas nuestras escuelas para interactuar con la era digital? La respuesta podría ser negativa, sin embargo, se tendría mayor certeza a partir de los resultados que arrojan las pruebas internacionales en las que participa Colombia.

Una de esas pruebas, el PISA ${ }^{12}$ (Program for International Student Assessment), en su versión 2009, puede aportar elementos que respondan la pregunta,

12 PISA es un estudio internacional comparativo de evaluación educativa liderado por la OCDE (Organización para la Cooperación y el Desarrollo Económico), que se realiza en ciclos trianuales en los que se evalúan estudiantes de 15 años, matriculados entre $7^{\circ}$ y $11^{\circ}$ grado, en las áreas de lectura, matemáticas y ciencias, con énfasis en una de éstas por ciclo. Colombia participó junto con 64 países en las pruebas 2009. a partir de los resultados que arroje el estudio sobre evaluación de la comprensión de texto digital por parte de nuestros estudiantes. Es importante señalar que esos resultados buscan aclarar las relaciones entre las destrezas y variables de tipo social, económico, demográfico, etc. Es decir, encontrar los indicadores de las características sociales que posee cada país participante (Gil, Guillermo).

Los retos para los participantes de dicha prueba se sintetizan en los siguientes aspectos: búsqueda y localización de información en Internet, utilización de hipertextos, lectura comprensiva, y construcción de pensamiento a partir de documentos electrónicos (El Tiempo). Sin embargo, la realidad colombiana en las escuelas muestra que tanto el acceso al uso de las TICs, como la comprensión y producción de texto electrónico, son aún limitados, por lo que no se espera que los alumnos obtengan resultados exitosos, por ahora.

La cuestión se complica debido a que algunas instituciones oficiales o privadas todavía no poseen aulas de informática, y otras, aunque cuentan con ellas, no han logrado las condiciones pedagógicas para un adecuado funcionamiento que facilite a los niños el desarrollo de un aprendizaje autónomo. En algunos casos carecen de la dotación suficiente para ubicar a un alumno por computador, por lo que deben trabajar dos o tres niños por máquina.

A la situación anterior se añade que no todos los equipos cuentan con acceso a Internet, y en algunos casos presentan mantenimiento deficiente (dispositivos descompuestos o limitado funcionamiento). Tampoco es evidente la democratización de la tecnología, pues fuera de la institución escolar muchos estudiantes no tienen acceso a la Internet, bien sea porque no tienen computador o porque carecen de recursos económicos para contratar un servicio de red.

En el caso de un amplio número de docentes, aún existe una separación entre la enseñanza en el aula tradicional y la que se encuentra dotada con tecnologías, pues si bien algunos interactúan con Internet y otros dispositivos, todavía no han logrado que el aprendizaje desde sus áreas se encuentre integrado con las TICs. 
Quedan pues las dudas acerca de los resultados de la prueba PISA, cuyo eje central es la comprensión de texto electrónico, a sabiendas de que en la actualidad la educación colombiana forma al niño y al joven en torno al texto impreso. Así mismo, queda planteado el desafío de la institución escolar para la preparación de sus alumnos a partir de ahora y en forma regulada, con el fin de que puedan responder exitosamente las diferentes pruebas relacionadas con la comprensión de texto digital. Familiarizarse con éste demanda una serie de destrezas no sólo manuales, sino cognitivas, que sólo se logran accediendo e interactuando con diversos dispositivos tecnológicos como las páginas Web, los juegos interactivos, los buscadores, los espacios de Chat y los buzones electrónicos.

No se descarta que al estudiante se le presenten retos como crear hipertextos, analizar contenidos de páginas Web y acceder a archivos gráficos, para determinar sus niveles de aprendizaje en torno a la lectura y escritura digitales. Así, los conceptos de información y conocimiento cambian ostensiblemente debido a la influencia de Internet y al grado de interactividad de los individuos.

Las TICs aparecen entonces como una alternativa para la renovación de las prácticas educativas en consonancia con la concepción y las estrategias pedagógicas, el modelo de enseñanza, el papel del maestro, los modos de aprendizaje del alumno, y la forma en que se organiza una clase. También se espera que con la facilidad que brindan las TICs se renueve la forma tradicional de tomar anotaciones en clase con el cuaderno y el bolígrafo y estos sean complementados por los dispositivos que permiten la producción de texto digital.

Dicha producción digital deberá facilitar a los interlocutores del proceso de aprendizaje un papel más interactivo con la discursividad virtual, una inmersión constructiva, y diversas posibilidades de creación, que vayan más allá del simple uso digital y permitan al usuario expresar refinados niveles de pensamiento creativo apoyado en las TICs.

La influencia de las TICs determina importantes cambios en las formas de comunicación y en los procesos de aprendizaje; en éstos últimos la lectura digital adquiere nuevas dimensiones de conceptualización y realización, por lo que debe analizarse desde otra perspectiva el rol de lector, como lo sugiere Eduardo Gutiérrez, para quien es necesario reformular la pregunta que surge cuando los procesos de lectura están fundamentados en las TICs: ¿cómo debe entenderse la lectura en estos días, cuando los avances tecnológicos están produciendo modificaciones en la idea que se tiene de géneros y narrativas cuyos espacios propicios son los formatos Hipermediales? (Gutiérrez, 2009, p. 146).

$\mathrm{Al}$ respecto se encuentran variadas respuestas, sin embargo es pertinente observar que en la actualidad se demanda a los estudiantes ser intérpretes de sistemas distintos de la escritura tradicional impresa, como la imagen, el sonido y el texto electrónico, condiciones que influyen en los cambios lectores y en su conceptualización. Por tanto ya no podría plantearse la lectura como una simple decodificación del código lingüístico, pues no basta con descifrar para leer, y tampoco es suficiente considerar dicho código como el único sistema de signos susceptible de ser leído; como señala Eduardo Gutiérrez, al hablar de lectura será necesario:

Localizar el contexto histórico, el régimen comunicativo y los sentidos dominantes socialmente; las prácticas de significación incluidas, las retóricas, formas narrativas y reglas de interacción de esa coyuntura; los tipos de lectores existentes y en formación, y las necesidades sociales de significado. [Así, la denominada práctica lectora puede ser concebida como una] [...] síntesis del conjunto de acciones y operaciones con las que un sujeto interactúa con un discurso construido, a partir de una serie de sistemas simbólicos y procesos de producción, de significación, y en el que intenta, más que llevar a cabo la comprensión como consumo de significado, insertarse en el flujo de producción social de sentido (Gutiérrez, 2009, p. 147).

Concebida de esa manera, la práctica lectora no se limita al sujeto, sino que se convierte en un hecho colectivo que, de acuerdo con su naturaleza, recurre a las comunidades y a los contextos culturales en los que este tipo de prácticas se realizan, de modo que el 
sujeto: "no es, ni puede ser un poseedor universal de la comprensión, sino que es, en concreto, parte de la dinámica de producción social" (Gutiérrez, 2009).

Ese proceso histórico muestra las transformaciones que está viviendo el alumno, entre las cuales se encuentran: la velocidad con que la información viaja hasta su destinatario o la rapidez con que es obtenida, la propuesta de otros lenguajes, la creación de dispositivos mejorados para procesar datos, la información difundida a través de los equipos móviles, o el libro digital compuesto por audio y video.

En el contexto anteriormente descrito surge ese lector contemporáneo que se encuentra inmerso en novedosas formas de creación o producción de significados dentro de la cultura. El libro-como se ha sugerido anteriormente-adquiere un nuevo soporte en la pantalla, y en consecuencia se debe modificar la manera en que se enseña a leer, lo que implica que el lector y la cultura de una sociedad requieran urgentemente acoplarse a los nuevos formatos.

Las formas de escritura tradicionales ya no satisfacen las exigencias del nuevo lector que busca otras experiencias y significados. La velocidad con que se obtiene la información es inmediata, el estudiante puede entrar en contacto diario con la televisión interactiva o la recepción de mensajes al celular, generando así una sociedad constante de usuarios lectores, que descubren formas de escritura en "un espacio hipernarrado" (Gutiérrez, 2009, p.154).

Para esta necesidad de acceder a la información se propone alfabetizar al joven a través de la lectura en formatos virtuales o multimedia, recurriendo a la opción de gráficos o videos debido a la rapidez con que estos se descargan. Otros recursos para desarrollar habilidades en la lectura o la escritura han sido las prácticas del Chat o messenger donde la comunicación se basa en el uso de emoticones o la simplificación de la escritura.

Esta interactividad en la red conduce al manejo de otros códigos y gramáticas propias de la escritura digital, y solamente conociéndolos se podrá acceder a significados que construye Internet, los cuales pueden ser modificados o corregidos por la intervención de un lector-escritor de formatos electrónicos.
Este trasfondo teórico y tecnológico subyace al proyecto de investigación antes mencionado, para el cual es fundamental la comprensión del momento cultural y de sus diversas formas y medios de aprendizaje, a partir de los cuales es posible hablar del desarrollo de la inteligencia individual y colectiva. Así mismo, deben replantearse los conceptos de comprensión, lectura, escritura, y el de interlocutor, entre otros, en el desarrollo de un proceso de aprendizaje, aspectos que han sido tenidos en cuenta para el desarrollo del proyecto.

\section{Los interlocutores contemporáneos}

El niño del siglo XXI requiere aprender en un espacio lúdico, no coercitivo, que le permita disfrutar de la literatura mientras juega; no es fortuita la mención al nuevo siglo, pues se ha caracterizado por avances en la digitalización y las tecnologías. Algunos de estos progresos han permitido cambiar la visión egoísta del aprendizaje como una tribulación y crear ambientes más amigables para los alumnos.

En las investigaciones adelantadas por el grupo HIMINI se ha podido observar que el estudiante de primaria disfruta del aprendizaje de la literatura gracias a su interacción con universos que fusionan la aventura y el conocimiento, apoyados en las TICs, como en el caso de los juegos interactivos y demás dispositivos ya mencionados.

Estas creaciones electrónicas familiarizan al niño en el contacto con imagen, texto, audio y diseño que encuentran complemento a través de Internet; también se ha observado que los estudiantes de primaria aprenden más placenteramente cuando la teoría se expresa mediante textos en formato electrónico, es decir, aquellos que brindan posibilidades de expansión de la información, son breves, con disposición interactiva y están diseñados especialmente para facilitar la inmersión de los usuarios.

En consonancia con los desarrollos de la discursividad contemporánea, que ha encontrado plena sintonía en los formatos electrónicos (Celular, blog, Ipod, Reproductores MP3, BlackBerry, etc.) gracias a la máxima concentración del lenguaje, también las creaciones literarias han asumido esta característica y revelan un significativo esfuerzo por parte de sus 
creadores al lograr las palabras justas para tejer un refinado arte verbal, tal como ocurre con el minicuento o la minificción.

A partir de esta última forma literaria se ha conseguido estimular el interés de los jóvenes lectores hacia la literatura, puesto que muchas de sus características han resultado de su agrado, tales como la brevedad de sus historias, la concisión, el humor, la ironía y la posibilidad de participar como cocreadores, entre otras. Las minificciones se adecuan por su forma a las tecnologías de nuestro tiempo, con las cuales ha ido creciendo el niño. La posibilidad que tiene el estudiante de aprender la teoría sobre el minicuento mediante el contacto directo con textos breves, apoyados en dispositivos tecnológicos, ha permitido el fomento de una atractiva e ingeniosa forma de aprendizaje en espacios novedosos como los ambientes Hipermediales.

Sin embargo, estas formas de promover el ingenio y la creatividad a partir de la comprensión y producción de textos breves, son escasas en las escuelas colombianas. Su ausencia es notoria al momento de evaluar los bajos niveles de lectura y escritura de los estudiantes, pues en la mayoría de instituciones de primaria todavía se registran muchas dificultades para la promoción de los procesos lecto-escritores en el ámbito literario, y en particular, de la minificción. Igual situación se presenta en otros espacios de formación escolar como los colegios de secundaria y las universidades.

El grupo investigador ha podido apreciar las limitaciones y carencias de los métodos que implementan las instituciones escolares con las cuales ha trabajado, aspecto que ha derivado en la abulia y el desinterés en el aprendizaje literario por parte de los estudiantes. A partir de dicha observación se consideró pertinente tener en cuenta los intereses de los niños y jóvenes para proponer prácticas pedagógicas novedosas y creativas que permitieran ambientes agradables para el aprendizaje de la literatura, la lectura y la escritura.

Los lectores contemporáneos que se encuentran en plena sintonía con una "cultura hegemonizada por el imperio de la imagen" y caracterizada por la liviandad, la rapidez, el aprendizaje lúdico y el dinamismo, expresan su necesidad de experimentar procesos de comprensión de texto que estén más en concordancia con la dinámica de su tiempo. Esto quiere decir, que les permita interactuar en ambientes de aprendizaje agradables fundamentados en discursos leves, amenos, humorísticos y sorprendentes, aspectos que ha considerado el aludido proyecto.

\section{Particularidades del proyecto}

El grupo HIMINI trabajó con los siguientes dispositivos tecnológicos que hacen parte del Ambiente Hipermedial para desarrollar el proyecto de investigación: un juego interactivo titulado El Damnum: la búsqueda del libro sagrado; una página Web con el nombre de El Portal de Galef; un blog denominado El Damnum; un buzón electrónico cuya dirección es actividadescarrasquilla@gmail.com al que los estudiantes remitieron sus inquietudes y respuestas relacionadas con los niveles, aventuras y retos del juego; también se crearon buzones personalizados por cada estudiante.

Complementariamente se utilizaron algunos buscadores de Internet como Google; mediante estas tecnologías se dio curso al uso de espacios mediáticos lúdicos e innovadores para facilitar el aprendizaje literario de los estudiantes de $5^{\circ}$ de primaria. Así mismo, se pudo analizar los efectos de dicho ambiente en el aprendizaje de los niños que interactuaron con él.

El conjunto de estos dispositivos tecnológicos, integrado dinámicamente en el Ambiente Hipermedial, adoptó como núcleo de aprendizaje la didáctica de la minificción y los procesos de comprensión y producción de texto literario breve digital. Con el propósito de promover un aprendizaje lúdico y formativo se seleccionó un grupo de estudiantes de grado quinto de primaria del Colegio Distrital Tomás Carrasquilla de la ciudad de Bogotá.

\section{Justificación}

La anterior panorámica de la lectura y la escritura en la educación colombiana, y las limitadas perspectivas de desarrollo de una educación literaria, indujeron al grupo HIMINI a indagar acerca de los 
espacios y condiciones más propicios para promover el aprendizaje de la literatura, en consonancia con los desafíos de la modernidad antes mencionados. Así, se encontró que como respuesta a dichas dificultades era pertinente recurrir a la creación de Ambientes Hipermediales que facilitaran el desarrollo de discurso estético en los estudiantes a partir de la comprensión y producción de textos breves.

Como núcleo de aprendizaje de dichos ambientes fue seleccionado el minicuento, género que ha hecho importantes aportes a los procesos literarios y se ha constituido en una de las formas contemporáneas más propicias para despertar el gusto por la literatura, la lectura y la escritura. Además, el discurso versátil que maneja facilita su interacción con otro tipo de discursos culturales y con diversos formatos tecnológicos.

Frente al divorcio entre el fomento de la literatura en la escuela y el empleo de las TICs, al que se suma el improvisado uso del computador, la respuesta sería un proceso de aprendizaje literario digital bien dirigido, que promueva unas actividades sistemáticas en las que sea posible integrar los textos literarios, las propuestas pedagógicas, y algunos dispositivos tecnológicos como juegos interactivos, páginas Web, blogs, procesador de texto, buscadores, buzones electrónicos y servicios de microblogging.

Dichas dificultades y expectativas pudieron verse superadas gracias a la implementación de un Ambiente Hipermedial como el creado por el grupo HIMINI, cuyos dispositivos facilitaron, entre otras, las siguientes ventajas: acceso rápido a las creaciones, disfrute de textos breves en versión digital con formatos especiales de diseño, conocimiento de una sencilla fundamentación teórica y desarrollo de un proceso de aprendizaje literario lúdico.

\section{Dispositivos tecnológicos del Ambiente Hipermedial}

La dinamicidad de dicho Ambiente Hipermedial, que ha facilitado el aprendizaje lúdico literario de los estudiantes, ha sido posible gracias a la integración de los dispositivos tecnológicos antes señalados, los cuales han desempeñado una función y permitido la interacción de los usuarios como se explica a continuación.

\section{Juego Interactivo El Damnum: la búsqueda del libro sagrado}

El juego denominado El Damnum: la búsqueda del libro sagrado, es un software lúdico-literario con fines didácticos, cuya ambientación épica gira alrededor de la minificción o minicuento. El usuario descubre varios niveles que se vuelven más complejos a medida que avanza en la historia y en su interacción con el juego; de esta manera experimenta un mayor nivel de inmersión, y los obstáculos, que inicialmente son enigmas, se convierten en retos que invitan a superarlos. Los progresos alcanzados por los jugadores indican el grado de apropiación conseguido mediante la comprensión y la producción textual.

El juego está compuesto de una introducción constituida por la historia del juego y seis nivelescon algunos subniveles-que se explican a continuación:

- Primer nivel: El "Bosque de los Enigmas"que incluye un subnivel llamado "El Jardín del Hada" - , aquí el usuario encuentra la posibilidad de un acercamiento preliminar a la lectura comprensiva y a la organización de minicuentos por medio de enigmas y retos.

- Segundo nivel: "Posada de los Tahúres"; despierta la capacidad de comprensión creativa que supera la etapa de interpretación literal y parcial. Inmersos los niños en el ambiente épico, asumen el rol del héroe que busca las claves, dialogando con diferentes personajes de la historia. Además se enfrentan a los desafíos de comprensión y producción textual que demandan los minicuentos incluidos en este nivel.

- El tercer nivel: "Gruta de los Pergaminos", facilita un diálogo con el "duende Nissim", quien guía al héroe hacia los desafíos que pronto surgirán. Aparecen entonces los pergaminos, cada uno con un minicuento que contiene un reto; de los nueve retos posibles, el estudiante- 
héroe solamente resolverá tres. Los retos tienen un mayor grado de dificultad en este nivel de comprensión de minicuentos, y los textos transmiten sentimientos y valores frecuentes: la esperanza, el deseo, el amor, etc.

- Durante el desarrollo de este nivel, el estudiante tiene la posibilidad de ampliar su experiencia como lector, interactuar con diferentes temáticas propuestas por la literatura, interpretar, analizar y realizar una comprensión creativa de lo narrado en cada historia. Las respuestas que producen los niños en este nivel están sujetas a un mayor esfuerzo en la comprensión y aproximación al discurso estético.

- El cuarto nivel: "Valle de la muerte"-que incluye el subnivel "Valle de la fantasía"-, es el espacio propicio para que el estudiante elabore sus propios minicuentos por medio de estrategias fantásticas; así, puede crear dos pequeñas historias donde la imaginación es esencial, pues debe dar nuevos finales a los minirrelatos, narrar desde la perspectiva de otros personajes, variar el sentido de la historia y dar continuidad a la misma.

- El quinto nivel titulado "Monte de los laberintos", permite al estudiante continuar con la invención de minicuentos inspirados en otros minicuentos. Algo importante en este nivel es la motivación de los jugadores porque están cerca del final del juego, donde derrotarán a las fuerzas del mal y obtendrán el libro de la sabiduría para salvar a la humanidad. Los estudiantes continúan apropiándose de estrategias de escritura y nociones teóricas para enfrentarse a la escritura de texto estético verbal.

- En el sexto nivel, “Cueva del dragón”, el héroe del juego (niño o niña) ya ha superado los obstáculos y se encuentra en el sitio donde se halla el "Libro Sagrado: El Damnum"; para obtenerlo deberá vencer al Dragón. Su reto no solamente es la batalla, sino escribir su mejor minicuento para recibir la clave de ingreso y otras armas necesarias para derrotar al enemigo, que es el hechicero Kadón. La invención del minicuento es de tema libre y puede ser elaborado con ele- mentos de animación facilitados previamente a los estudiantes.

\section{Página Web: El Portal de Galef}

Otro dispositivo tecnológico del Ambiente de Aprendizaje fue una página Web denominada $E l$ Portal de Galef, que permite, a través de los distintos enlaces, el acceso a la información teórica necesaria sobre el minicuento, a una relación de direcciones electrónicas relacionadas con el tema, a un foro, y una antología de minicuentos de autores reconocidos y de estudiantes. Los vínculos que posee este portal aportan informaciones sobre los componentes del juego y el Ambiente, sus generalidades, descripción de cada nivel, misión de cada personaje, teoría del minicuento y direcciones relacionadas con este género discursivo.

\section{Blog El Damnum}

Este dispositivo tecnológico permite una interacción enriquecedora entre los estudiantes que fungen como héroes del juego y el sabio Galef, quien es el profesor. Mediante este el sapiente anciano se da respuesta a las creaciones textuales de los héroesestudiantes, se les propone tareas y se orientan sus actividades. La interacción de los usuarios no sólo es conocida por ellos, sino por quienes acceden al $b l o g$, cuya visibilidad es propia de un espacio de esta naturaleza ubicado en Internet.

El blog presenta imágenes atractivas de guerreros, hechiceros, y otro tipo de personajes conocidos por los niños, además incluye dos antologías de minicuentos a partir de las cuales se han desarrollado actividades de comprensión de lectura y un taller. También tiene otras etiquetas, con alguna explicación breve dirigida a los héroes.

En el encabezado del blog se explica que dicho espacio tecnológico ha sido creado con el propósito de intercambiar experiencias de lectura y escritura literarias, como complemento de la interacción de los estudiantes de educación básica primaria con un Ambiente Hipermedial producido por el grupo HIMINI.

Además de las etiquetas, el blog posee una entrada de Twitter, servicio gratuito de microblogging, 
desde donde el Sabio Galefinforma brevemente a los héroes acerca de sus avances en la aventura y reporta quiénes son los más adelantados en el juego. Uno de los contenidos presentados es el siguiente:

Los valientes guerreros Felipe y Juan José ya han llegado al Valle de la Muerte y están luchando por salir. ¿Quiénes serán los próximos?

También por medio de Twitter, Galef anima a los guerreros a avanzar con más rapidez por el juego para lograr el rescate del Libro Sagrado, un ejemplo de ello es el siguiente mensaje:

Valientes guerreros, soy el Sabio Galef. Quiero alertarlos para que se apresuren: ¡El malvado Kadón se acerca peligrosamente al Damnum!

Finalmente, el sabio Galef agradeció a través de Twitter la participación de los niños con el software diseñado por el grupo HIMINI: “Guerrer@s, gracias por su interacción con el juego "El Damnum: la búsqueda del Libro Sagrado". Su sabiduria los ha llevado lejos. Sabio Galef".

Una breve descripción del $b l o g$ permite apreciar el contenido de sus etiquetas así:

- La primera es una etiqueta de presentación llamada "Hola", que da la bienvenida a los estudiantes

- La segunda etiqueta se titula "Presentación del grupo HIMINI”, aquí se muestra el perfil del Grupo de Investigación en Hipermedia, Minificción, Literatura y Lenguaje HIMINI, y quiénes son sus integrantes y auxiliares.

- La tercera se conoce como "Antologías", y se divide en dos partes: "Pequeñas historias para valientes guerrer@s” “"Lecturas para los valientes guerrer@s”, las dos presentan breves textos acompañados de imágenes alusivas a cada historia. Algunos autores con creaciones incluidas son: Alexandra David-Neel, Elkin Obregón, Jairo Aníbal Niño, Claudio de Castro, Héctor Rojas Herazo, Braulio Llamero.

- La cuarta etiqueta se llama "Direcciones relacionadas con la literatura”, allí los estudiantes pueden explorar algunos links para conocer textos de páginas Web sobre algún tema literario.

- La quinta etiqueta se conoce bajo el nombre de "Direcciones sobre minificción”, y está constituida por links o direcciones electrónicas que ofrecen la posibilidad al estudiante de conocer otros sitios de Internet donde puede ampliar sus conocimientos sobre minificción.

- La sexta etiqueta: "El Ambiente Hipermedial del grupo HIMINI", hace una presentación de los dispositivos tecnológicos que componen el Ambiente, destinados al impulso del aprendizaje literario en niños de quinto de primaria.

- La séptima etiqueta es "Eventos que realiza el grupo HIMINI", y brinda información sobre el VI Congreso Internacional de Minificción a través de su primera circular. También se mencionan algunas conferencias organizadas por la Universidad Pedagógica y el HIMINI como antesala al "Primer Encuentro Nacional de Minificción "Luis Vidales" realizado el año anterior.

- En la octava etiqueta: "Otros blogs de interés literario", los niños pueden explorar diferentes espacios tecnológicos sobre literatura en formato de blog.

- La novena etiqueta lleva el título de "Publicaciones", allí el estudiante puede acceder a información sobre textos relacionados con la minificción en Colombia, el minicuento en la literatura colombiana y la serie La Avellana.

- La décima etiqueta, "Recomendaciones del sabio Galef", presenta un mensaje escrito por el anciano para aquellos que ingresan a la $\mathrm{Po}$ sada de los Tahúres, además de las indicaciones que le recuerdan al héroe leer detenidamente cada instrucción y analizar con cuidado las pequeñas historias para acceder al sentido que encierran, el sabio les pide tener en cuenta el parlamento de cada uno de los personajes que se encuentra en dicho nivel del juego interactivo.

- La última etiqueta se presenta como las "Respuestas del sabio Galef a Luis Felipe y Daniel”, 
y es un ejemplo de cómo el sabio interactuaba con los alumnos, revisando las respuestas a los retos, sugiriéndoles amablemente correcciones, guiándolos hacia la solución acertada y animándolos mediante la motivación a continuar en el desarrollo del juego.

\section{Buzones electrónicos}

Los buzones electrónicos son importantes porque permitieron a los niños escribir, envíar sus textos y recibir retroalimentación en el desarrollo de los diferentes retos que propone el juego a partir del segundo nivel (Posada de los Tahúres). Los escritos fueron remitidos a la dirección electrónica (actividadescarrasquilla@gmail.com) perteneciente al sabio Galef (grupo de investigación), quien corrige amablemente el trabajo elaborado por los alumnos, felicita sus progresos, y anima a los jugadores a seguir adelante en su misión. Las respuestas de Galef fueron remitidas en archivos adjuntos que luego recibieron los estudiantes en los buzones creados por el Grupo HIMINI para cada pareja de alumnos.

Los estudiantes leían las sugerencias del sabio desde sus correos a través de dos opciones: "Visualizar como HTML" o "Descargar", posteriormente llevaban a cabo las recomendaciones para mejorar sus respuestas a los retos o actividades elaboradas para cada sesión. Gracias a los buzones se pudo complementar la comunicación con los estudiantes y cumplir con una adecuada retroalimentación.

\section{Buscadores}

Buscadores como Google y Altavista fueron de gran utilidad para la localización de El portal de Galef, el blog El Damnum, y otras páginas relacionadas con estudios, creación e investigación sobre el minicuento, tales como http://www.literaturas.com, de gran utilidad para el tema del microrrelato, a partir de las cuales se pudo realizar un taller sobre texto digital para mejorar el proceso lector hipertextual estudiantil; Google sirvió también para que los estudiantes aprendieran a localizar imágenes en Internet, que posteriormente copiaban y pegaban en el texto digital que diseñaron en el procesador de texto Word.

\section{Páginas Web}

El número de dispositivos tecnológicos señalados se complementa con el uso de páginas Web en las que los niños exploran informaciones teóricas sobre la minificción a partir de los textos de especialistas del tema. Por ejemplo, en una de las sesiones se trabajó el texto electrónico de Dolores Koch, Algunas ideas sobre la minificción, localizado en la dirección http:// www.literaturas.com/doloreskorscks.htm. De este modo se complementó el estudio teórico de los niños sobre este género y también ayudó al proceso de habituarse a la lectura de textos teóricos breves frente a la pantalla del computador.

\section{Papel del estudiante}

Ha sido interesante ver la forma en que el estudiante interactúa con el juego, desarrolla destrezas a través de su inmersión en la aventura, maneja la información de Internet para ingresar al Portal de Galef, y descubre las pistas que necesita para pasar a otros niveles dentro del juego.

Dichas pistas son respuestas a preguntas que los personajes del juego hacen al usuario acerca del minicuento. La necesidad de seguir dentro del juego hace que el niño busque el conocimiento sobre este tipo de texto; poco a poco el alumno va haciendo uso de la selección de la información, las inferencias, la organización textual y la eliminación de otras informaciones hasta obtener un texto con sentido pertinente.

En otras palabras, mientras el estudiante juega e interactúa con los dispositivos tecnológicos, desarrolla destrezas creativas, accede a la información necesaria de carácter literario y utiliza su buzón electrónico para enviar ejercicios de producción textual al correo del sabio Galef (personaje guía de los héroes del juego), quien contesta a cada usuario, y lo lleva por el sendero del aprendizaje, mostrándole los aciertos y desaciertos que ha obtenido frente a los retos propuestos.

El alumno adquiere un rol activo como participante de la trama, así se aleja de la abulia que muchas veces implica, con el uso de herramientas tradicionales, la clase de literatura. Con la propuesta del grupo HIMINI el héroe-estudiante asume la mi- 
sión de obtener el Damnum o libro de la Sabiduría, para lo cual debe vivir aventuras en lugares rodeados de enemigos, animales feroces, un enfrentamiento final con el hechicero Kadón y simultáneamente, el desarrollo de su discurso estético mediante la comprensión y producción de textos con cierto nivel estético.

\section{Papel del maestro}

En la aplicación del Ambiente Hipermedial el docente está llamado a desempeñar una importante labor para garantizar una fértil interacción de los estudiantes con los dispositivos tecnológicos. Con tal propósito, debe conocer previamente el funcionamiento del Ambiente, sus dispositivos tecnológicos, y dominar la teoría sobre el tipo de texto artístico conocido como minicuento. Complementando esto, el profesor puede recurrir a estrategias pedagógicas para desarrollar la comprensión y producción de textos digitales breves y minicuentos durante la clase y a través de la elaboración de talleres.

Cuando se inicia el trabajo con el Ambiente, el maestro se convierte en otro actor del juego que acompaña constantemente los desafíos de los estudiantes en su interacción con los dispositivos tecnológicos; en su función de sabio, Galef debe estar pendiente de las preguntas más comunes que surgen de la interacción de los estudiantes con el Ambiente, y en particular, con el juego interactivo. A partir del segundo nivel su función se hace más protagónica, pues además de orientar discursivamente al héroe, debe efectuar una labor especializada de retroalimentación para garantizar el perfeccionamiento paulatino de los estudiantes en sus procesos de comprensión y producción textual.

Además de su labor discursiva retroalimentadora, que se produce vía Internet, la función del profesor en su rol de Galef aporta a los estudiantes-héroes otros conocimientos, relacionados con el diseño de textos digitales mediante el procesador de Word y el uso de otros dispositivos tecnológicos como el buzón electrónico, el blog y los buscadores. También facilita información recurriendo a los servicios de microblogging (Twitter).
A diferencia de otros dispositivos tecnológicos, que sólo comprometen a los estudiantes y excluyen al profesor, el Ambiente Hipermedial propuesto por el grupo HIMINI, vincula por igual en una labor creativa a los dos, pues el maestro se ve en la necesidad de fungir como sabio Galef para estar a tono con las demandas de los estudiantes a partir de su interacción con el juego. Como ya se ha señalado, el acompañamiento retroalimentador del docente es fundamental para el progreso discursivo de los estudiantes, sin embargo, el éxito de dicha actividad será relativo si no está mediado por un constante estímulo del profesor para el avance de los jugadores.

Pese a que el estudiante adquiere paulatinamente un importante grado de autonomía a medida que avanza en su interacción con el Ambiente, el maestro sigue estando presente en el acompañamiento, pues además de que asume diferentes roles de acuerdo con el nivel del juego en que se produce su retroalimentación, debe continuar hasta el final el análisis individual y colectivo de los procesos de interpretación literaria y de escritura creativa que producen los niños en su aventura con el juego.

Aunque los héroes tengan mucho entusiasmo para enfrentar la aventura del juego y el desafío discursivo, es frecuente encontrar en sus respuestas algunas limitaciones de escritura y de creación de sentido, razón por la cual se hace cada vez más importante el profesor o sabio Galef para ayudarlos a perfeccionar su discurso con sus sapientes recomendaciones, solicitándoles amablemente reescribir el texto, corregirlo, ampliar su solución, mejorar su redacción o producir un escrito mucho más claro.

Así, el docente desempeña dos roles: en el aula de clase es el orientador del aprendizaje individual y grupal en relación con las diferentes áreas, incluida la asignatura de Lengua Castellana, y en el aula de informática es el sabio Galef, quien se comunica con los estudiantes-héroes mediante los buzones electrónicos con el fin de contribuir al mundo imaginario y a los desafíos discursivos que propone el juego interactivo.

En su condición de sabio, el profesor se ve en la necesidad de adoptar un tono de consejero para guiar a los héroes y recordarles que deben cuidarse 
y apresurarse en la búsqueda del Libro Sagrado. Además, les advierte sobre los peligros a que están expuestos con los Tradoths, servidores del hechicero Kadón, quienes pueden atraparlos si no están atentos en su desplazamiento por los diferentes espacios de la aventura. Incluso, podrían "perder la vida" y verse obligados a comenzar de nuevo, recorriendo el primer nivel, si sus carencias, tanto en sus desplazamientos por el juego como en sus respuestas a los retos son considerables.

Los consejos, respuestas y sugerencias que brinde el profesor directamente, o a través de Galef, necesitan creatividad, claridad, y exactitud para garantizar el progreso discursivo estudiantil. Además, deben motivar al estudiante a seguir con entusiasmo y gran atención por los niveles del juego y las respuestas a los retos; $y$, como la labor de seguimiento exige tener presentes los logros y dificultades grupales e individuales, el docente necesita llevar un diario donde registre todo lo que ocurre en cada sesión, para, a partir de dicha información, crear estrategias y solucionar inconvenientes que no estén permitiendo el aprendizaje o la evolución de los héroes con el Ambiente.

Como complemento, debe proponer otras actividades que estimulen a los niños y les permitan encontrar nuevos horizontes en sus búsquedas narrativas breves. Concluida la aventura, premiará a los que ocupen los primeros lugares con un juguete alusivo a la historia épica, por ejemplo, la espada de la luz; a los demás héroes o competidores también se les reconocerá su esfuerzo con algún presente (esferos, lápices, dulces, galletas, etc.), así los héroes no sentirán que estuvieron dentro de una clase tradicional, sino en una verdadera aventura, en la que a la vez que jugaron y recorrieron lugares fantásticos, se acercaron al minicuento y aprendieron de éste en forma lúdica.

\section{Trasfondo teórico del proyecto}

El Ambiente de aprendizaje se fundamentó en las siguientes perspectivas teóricas: Didáctica de la literatura orientada hacia narrativas breves, específicamente el Minicuento, la Minificción Digital, el poema lírico y en prosa, las fábulas clásicas y contem- poráneas, rimas, acrósticos, minitextos y horóscopos; también abordó el tema de lectura y escritura en entornos digitales; la teoría literaria de los estudios dialógicos; los análisis e investigaciones acerca del impacto de dispositivos tecnológicos digitales con fines educativos (Páginas Web, software educativo, Ambientes Hipermediales, blogs, microblogging, procesador de texto Word, buzones electrónicos y buscadores).

Desde la didáctica de la literatura y el minicuento, se mantuvieron las propuestas de Italo Calvino (1998), quien encuentra que la suerte de la literatura y del libro en la era tecnológica, no puede desconocer elementos propios de nuestro tiempo como la rapidez y la concisión, porque la literatura es eficaz en la brevedad si se une a formatos electrónicos.

El grupo HIMINI ha venido constituyendo, a través de sus investigaciones, propuestas didácticas que tienen como núcleo teórico y práctico el minicuento y la minificción en entornos digitales. Este tipo de discursos artísticos verbales se trabajaron en el proyecto desarrollado, apoyados en el Ambiente Hipermedial descrito anteriormente; este proceso no solamente promovió la lectura de textos breves electrónicos, sino la producción de cuentos cortos por medio del uso de dispositivos como el procesador de texto Word y sus herramientas, que permitieron la creación de textos digitales creativos.

Uno de los documentos teóricos en los que se fundamentó el proyecto ha permitido argumentar la necesidad de propiciar un Ambiente de aprendizaje centrado en la Minificción Digital, cuyo uso refuta la creencia de que la televisión y el computador hacen que los niños y jóvenes lean en menor cantidad. Como señala su autora, la especialista Roxana Morduchowicz (2008), suele ocurrir lo contrario, es decir, que los niños y jóvenes establezcan un diálogo complejo con otros formatos; de la misma forma, se puede presentar un "efecto acumulativo" (Morduchowicz, 2008, p. 90), una actitud mediante la cual el niño y el joven conviven con muchas tecnologías y añaden a su interacción otras que aparecen con el tiempo.

En consonancia con la corriente literaria denominada "estudios dialógicos", inspirada en el pensa- 
dor Mijail Bajtin, se consideraron los tres elementos fundamentales que dicho autor señala para la estructuración artística del texto literario: el autor, el texto mismo y el lector, cada uno contribuyendo con la fina urdimbre del diálogo estético que caracteriza los procesos de producción y recepción de la obra. El diálogo que generó el proyecto, vinculó como interlocutores a los niños, quienes se familiarizaron con algunos autores de la minificción o de historias breves, mediante su interacción con los dispositivos tecnológicos.

Aunque existen antecedentes de la relación entre literatura y tecnología, es en nuestro tiempo cuando es posible aceptarla como una realidad. Alain Vuillemin (2005, p.169) menciona que en 1979 existían "aproximaciones animadas" de poemas visuales a través del ordenador que no tuvieron la debida recepción; posteriormente se hablaría de una poesía sonora (Vuillemin, 2005, p. 174), porque el sonido puede convivir con la imagen a través de una propuesta multimedia, pero será en 1989 cuando surge la primera revista regular de poesía electrónica.

La propuesta constituida por los anteriores referentes teóricos contempló tres momentos:

1. Diálogo inicial de creación estética entre los sujetos, para reconocer las voces presentes en sus discursos previos a la interacción con el Ambiente Hipermedial, mediante instrumentos reflexivos.

2. Diálogo de interacción entre el sujeto y el Ambiente Hipermedial, para producir réplicas a los discursos estéticos digitales propuestos.

3. Reconocimiento de nuevas voces en la producción del discurso estético electrónico de los sujetos.

\section{Recolección de información}

Los instrumentos diseñados para la recolección de la información fueron:

- Encuestas tecnológicas.

- Encuestas de lecto-escritura.

- Talleres de exploración literaria.
- Talleres integrados de comprensión y producción de texto digital y minicuento.

- Diarios de campo.

\section{Resultados}

Los resultados de esta experiencia permitieron identificar problemas en las prácticas pedagógicas actuales, especialmente en el área del lenguaje y literatura, pues aún persisten enfoques centrados en la alfabetización técnica que olvidan los procesos de comprensión y producción de textos basados en situaciones, necesidades e intereses reales de los estudiantes. Además, son pocos los trabajos de investigación orientados a la institución escolar que exploren nuevos horizontes didácticos en los que se vinculen las TICs y las diversas áreas de aprendizaje, para la innovación de los procesos educativos.

Pueden señalarse otros resultados como los siguientes:

1. La interacción de los niños con el Ambiente Hipermedial permitió una enriquecedora aproximación al minicuento digital, prueba de ello, son los múltiples textos de comprensión y producción relacionados con este género que remitieron al buzón electrónico del proyecto.

2. Las producciones creativas realizadas por los estudiantes, luego de su interacción con el Ambiente, mostraron significativos logros en el desarrollo de competencias discursivas, creativas y argumentativas al momento de crear textos narrativos breves.

3. La aplicación del Ambiente Hipermedial no sólo permitió reducir la brecha digital mediante el uso instrumental del computador, sino que proyectó a los estudiantes a un conocimiento de segundo grado, gracias a la interacción que tuvieron con dispositivos tecnológicos más complejos. Así, se pudo observar el desarrollo de destrezas tecnológicas relacionadas con los juegos interactivos, el uso de buzones electrónicos y de blogs.

4. A diferencia de años anteriores en que apenas un reducido número de estudiantes tenía 
conocimiento básico del computador y de Internet, en esta ocasión se pudo observar que el número ha crecido y, luego de su interacción con el Ambiente, se ha ampliado su conocimiento sobre las opciones que brinda el procesador de texto Word como: "abrir documento", "guardar", "minimizar", "restaurar", "diseñar página”, "fondo y bordes", "modificación de fuente", etc.

5. Además, los estudiantes aprendieron a utilizar recursos de Internet como: buzones electrónicos para escribir, enviar y abrir mensajes; descargar archivos adjuntos, visualizarlos o remitir sus trabajos como adjuntos. Algunos estudiantes también utilizaron con facilidad el buscador Google para localizar el blog El Damnum, el Portal de Galef, o para acceder a la opción de imágenes, que utilizaron en sus textos.

6. Los trabajos realizados por los estudiantes fueron significativas aproximaciones a la comprensión y producción de minicuento digital, utilizando para ello, texto, imagen, y diseño electrónico. Además, recurrieron al blog como medio de comunicación para la lectura de minificciones digitales y como medio orientador para la realización de talleres. Complementariamente utilizaron los buzones para enviar archivos adjuntos con las creaciones breves que produjeron.

7. En su interacción con el Ambiente, los estudiantes participaron en dos tipos de diálogo: el primero, con el sabio Galef mediante el juego interactivo; y el segundo, a través de Twetts ubicados en el $b l o g$, por medio de los mensajes del mencionado sabio (o investigadores del grupo HIMINI) remitidos a los buzones estudiantiles a manera de retroalimentación.

8. El alumno accedió a diversos textos breves literarios y no literarios desde un entorno digital, y su lectura adquirió nuevas características, diferentes de las que se logran con el material impreso, pues al hacer uso del mouse, de los links, y los contenidos de los múltiples entornos digitales, pudo experimentar su accesibilidad a amplios inventarios de información y la rapidez en el conocimiento de estos. Además, encontró audio, imágenes y textos cuya versatilidad en el uso le hicieron posible sus producciones diseñadas.

\section{Dificultades}

Si bien es cierto se registran los resultados del proyecto a manera de logros alcanzados, también es importante señalar algunas dificultades experimentadas en desarrollo del mismo:

1. Las dificultades de los alumnos para comprender los minicuentos digitales, o para elaborar sus propios textos breves electrónicos, reflejaron el reducido tiempo que tienen para interactuar a diario en la escuela, o fuera de ella, con la lectura y con algunos desarrollos de las TICs, como los procesadores de texto, Internet, algunos dispositivos tecnológicos de uso común (por ejemplo los juegos interactivos), buscadores o blogs.

2. Aunque los niños se acercaron a la producción del texto breve digital (fábulas, minicuento, minificción, minitexto, etc.), aún necesitan la orientación del profesor (o investigador) para perfeccionar sus creaciones.

3. El tiempo limitado del que dispusieron para interactuar con el Ambiente Hipermedial en el aula de sistemas impidió mayores avances en la elaboración de textos breves electrónicos. Las sesiones de comprensión de textos breves digitales aún deben trabajarse con los niños, pues los alumnos deben practicar más la lectura, análisis e interpretación de este tipo de textos. A pesar de algunas limitaciones, al comparar los resultados en algunas etapas del proceso, se observaron significativos avances en la comprensión y producción de textos con intención artística realizados por los niños.

4. A pesar de que algunos estudiantes-héroes lograron alcanzar el subnivel "Valle de la Fantasía” gracias a sus progresos en la producción 
de minicuento digital, se pudo observar que algunas de sus respuestas requieren todavía cierto nivel de perfeccionamiento, es decir, deben corregir la estructura lingüística y el estilo narrativo. Es importante señalar que algunos estudiantes accedieron al mismo sin seguir el orden de los niveles del juego, es decir, llegaron a este subnivel sin pasar por el anterior, de tal modo que dejaron algunos retos sin solución.

5. Algunos alumnos no lograron habituarse a la lectura digital, esto se debió a la falta de atención, y al poco tiempo del que disponen en la escuela para trabajar conjuntamente sus materias con las TICs. La escuela y otras instituciones educativas en Colombia necesitan innovar, en poco tiempo, el aprendizaje por medio de tecnologías vigentes y propuestas pedagógicas novedosas, que mantengan una comunicación con Internet, campus virtuales, textos electrónicos de estudio, páginas Web, aulas de clase inteligentes, $\mathrm{y}$ un personal docente preparado para interactuar en estos espacios, además de una alfabetización donde el alumno aprenda cómo leer en el contexto digital.

6. Una situación importante de señalar es la que descubre que mientras se observan progresos en el nivel de alfabetización básica en informática de los estudiantes, también prevalece algún divorcio entre los maestros, especialmente en la relación de su especialidad con las TICs.

Finalmente, es necesario destacar que en el panorama contemporáneo se requiere con urgencia una educación más flexible, con nuevas formas de enseñanza vinculadas a las TICs, y una práctica pedagógica innovadora que reconozca las ventajas que aportan los desarrollos tecnológicos a los procesos de aprendizaje de las diferentes áreas y en particular a la literatura.

Al considerar relevante estas formas y prácticas educativas, no se pretende desconocer o excluir a las demás, muchas de las cuales se fundamentan en acciones ingeniosas de los maestros, cuyos materiales básicos son los textos impresos, el lápiz y el papel. Sin embargo, creemos que en un mundo cambiante donde las Tecnologías de la Información y la Comunicación vienen desempeñando un rol cada vez más protagónico, no es conveniente desconocer estos avances, satanizarlos, o declararlos la única opción en cuestiones de innovación pedagógica, pero si ubicarlos en su justa dimensión como valiosos apoyos para el progreso de un aprendizaje lúdico en el ámbito literario.】 


\section{Bibliografía}

Bajtin, M. (1997). 'La palabra en la vida y la palabra en la poesía. Hacia una poética sociológica'. Hacia una filosofía del acto ético. Barcelona: Anthropos.

Borrás Castanyer, L. (2005). Textualidades electrónicas. Nuevos escenarios para la literatura. Barcelona: Editorial UOC.

Bustamante, E. (2004). Comunicación y cultura en la era digital. Industrias, mercados y diversidad en España. Barcelona: Gedisa.

Brown, E., y Chignell, M. (1993). 'Learning by linking: pedagogical environments for hypermedia authoring. Journal of computer in higher education, v. 5, $\mathrm{N}^{\circ} .1,27-50$.

Bustamante, G., y Kremer, H. (1994). Antología del cuento corto colombiano. Cali: Universidad del Valle.

Calvino, I. (1989). Seis propuestas para el próximo milenio. Madrid: Siruela.

Coovert, R. (1992). The End of Books. Extraído el 27 de Julio de 2010, desde http://wings.buffalo.edu/english/ faculty/conte/syllabi/370/EndofBooks.htm

Cruz, H. (1997). 'Diseñando aplicaciones de realidad virtual para el desktop'. Revista Interamericana de Nuevas Tecnologías de la Información, v. 3, Nº 1 y 2.

De Haro, J. J. 'Microblogging para la educación'. Educativa. Obtenido el 5 de octubre de 2009, desde http:// jjdeharo.blogspot.com/2009/08/microbloggingpara-la-educacion.html

Do Brito, M. (2010). Umberto Eco, la cultura alfabética y la cultura de la imagen. Extraído el 26 de julio de 2010, desde http://marisdobrito.blogspot.com/2010/04/ umberto-eco-la-cultura-alfabetica-y-la.html

Eco, U. (1996). Seis paseos por los bosques narrativos. Barcelona: Lumen.

Eduteka (2007). El porqué de las Tic en educación. Extraído el 5 de octubre de 2009, desde http://www. eduteka.org/PorQueTIC.php

El Tiempo (2009). ¿Sí está preparada la escuela de Colombia para la era digital? Obtenido el 27 de Julio de 2010, desde http://www.mineducacion.gov.co/ observatorio/1722/article-183070.html

Frechín, J. Tweetboard. Otra forma de intergrar twitter en tu web. Extraído el 1 de octubre de 2009, desde http://www.tunelcarpiano.net/2009/06/tweetboardotra-forma-de-integrar.html
Genette, G. (1989). Figuras III. Barcelona: Lumen.

Gil, G. El proyecto P.I.S.A de la OCDE. Extraído el 26 de julio de 2010, desde http://www.ince.mec.es/pub/ pisa.htm

Gutiérrez, E. (2009, enero-junio). 'Leer digital: la lectura en el entorno de las nuevas tecnologías de la información y la comunicación'. Signo y pensamiento, v. XXVIII, 145-163. Bogotá: Pontificia Universidad Javeriana.

Landete, S. (2009). Diez formas de usar Twitter en el aula. Extraído el 5 de octubre de 2009, desde http://www. tecnotic.com/node/746\#

Landow, G. (1995). Hipertexto: La convergencia entre la teoría crítica contemporánea y la tecnología. Barcelona: Paidós.

Landow, G. (1997). Teoría del hipertexto. Barcelona: Paidós.

Lopez, J. M. (2010). Tweetie. Extraído el 1 de octubre de 2009, desde http://tweetie.softonic.com/mac

Mc-Laughin, B. (1991). Theories of Second Language Learning. London: Edward Arnold.

Mc-Clintock, R. (1992). Power and Pedagogy: Transforming Education through Information. Extraído el 27 de Julio de 2010, desde http://www.ilt.columbia.edu/ publications/Projects/digitexts/mcclintock/title.pdf

Morduchowicz, R. (2008). La generación multimedia. Significados, consumos y prácticas culturales de los jóvenes. Buenos Aires: Paidós.

Muñoz, J. Enseñando con Twitter. Extraído el 5 de octubre de 2009, desde http://clarion.mudejarico.es/ archives $/ 148$

Murray, J. H. (1999). Hamlet en la holocubierta. Barcelona: Paidos.

Oxford, R. (1990). Language learning Strategies: What every teacher should know. Boston: Heinie \& Heinle publishers.

Pennac, D. (1992). Como una novela. Bogotá: Norma.

Pérez Beltran, Á. M. (1997). Cuento y minicuento. Bogotá: Página Maestra Editores.

Pollastri, L. (1994). Una escritura de lo intersticial: las formas breves en la narrativa hispanoamericana contemporánea. El puente de las palabras. Homenaje 
a David Lagmanovich. Washington: OEA- INTERAMER.

Ricoeur, P. (1980). La metáfora viva. Madrid: Ediciones Cristiandad.

Rodari, G. (1996). Gramática de la fantasía. Barcelona: Ediciones del Bronce.

Rodríguez Romero, N. (1996). Elementos para una teoría del minicuento. Tunja: Colibrí Ediciones.

Tomassini, G., y Maris Colombo, S. (1998). Comprensión lectora y producción textual, Minificción hispanoamericana. Rosario: Fundación Ross.

TweetDeck, la mejor opción para Twittear. Extraído el 1 de octubre de 2009, desde http://ardetroya.com. ar/1739/tweetdeck-la-mejor-opcion-para-twittear. html

Tweeterrific. Extraído el 5 de agosto de 2009, desde http:// bitacoras.com/canales/twitterrific
Vouillamoz, N. (2000). Literatura e hipermedia. Barcelona: Paidos.

Wikipedia. Facebook. Obtenido el 1 de octubre de 2009 desde http://es.wikipedia.org/wiki/Facebook.

Wikipedia. Twinkle. Obtenido el 1 de octubre de 2009 en http://es.wikipedia.org/wiki/Twinkle.

Wikipedia. Web 2.0. Obtenido el julio de 2009 desde http://es.wikipedia.org/wiki/Web_2.0\#Introducci. C3.B3n

Wikipedia. Web 3.0. Extraído el 2 de julio de 2009 desde http://es.wikipedia.org/wiki/Web_3.0

Zavala, L. (1999). Lecturas simultáneas: la enseñanza de lengua y literatura con especial atención al cuento ultracorto. México: Universidad Autónoma Metropolitana.

Zavala, I. M. (1991). La posmodernidad y Mijail Bajtín. Una poética dialógica. Madrid: Espasa Calpe. 